Published in final edited form as:

J Aircr. 2017 November ; 54(6): 2367-2377. doi:10.2514/1.C034407.

\title{
Flight Test Techniques for Quantifying Pitch Rate and Angle of Attack Rate Dependencies
}

\author{
Jared A. Grauer ${ }^{\star}$, Eugene A. Morelli ${ }^{\dagger}$, Daniel G. Murri ${ }^{\ddagger}$ \\ *Research Engineer, Dynamic Systems and Control Branch, MS 308. Senior Member AIAA, \\ NASA Langley Research Center, Hampton, Virginia 23681. \\ ${ }^{\dagger}$ Research Engineer, Dynamic Systems and Control Branch, MS 308. Associate Fellow AIAA, \\ NASA Langley Research Center, Hampton, Virginia 23681. \\ ¥NASA Technical Fellow for Flight Mechanics, NASA Engineering and Safety Center, MS 308, \\ Associate Fellow AIAA, NASA Langley Research Center, Hampton, Virginia 23681.
}

\begin{abstract}
Three different types of maneuvers were designed to separately quantify pitch rate and angle of attack rate contributions to the nondimensional aerodynamic pitching moment coefficient. These maneuvers combined pilot inputs and automatic multisine excitations, and were demonstrated with the subscale T-2 and Bat-4 airplanes using the NASA AirSTAR flight test facility. Stability and control derivatives, in particular $C_{m_{q}}$ and $C_{m_{\dot{\alpha}}}$, were accurately estimated from the flight test data. These maneuvers can be performed with many types of aircraft, and the results can be used to improve physical insight into the flight dynamics, facilitate more accurate comparisons with wind tunnel experiments or numerical investigations, and increase simulation prediction fidelity.
\end{abstract}

\section{Introduction}

THE pitch rate of an aircraft is a rate of change of the aircraft orientation with respect to an inertial frame, expressed in body axes. The angle of attack rate is a rate of change of the aircraft orientation with respect to the air-relative velocity. Arbitrary motion in flight can involve one, both, or neither of these rates. For relatively small perturbations, reduced frequencies, and Mach numbers, the effects of angular velocities on the aerodynamic forces and moments acting on the aircraft can be modeled using conventional stability derivatives, after Bryan. ${ }^{1}$ The largest contributions from these rates are on the nondimensional pitching moment coefficient, and are represented by the parameters $C_{m_{q}}$ and $C_{m_{\dot{\alpha}}}$. The former is usually attributed to the additional lift acting on the horizontal tail during rotation in pitch, whereas the latter is an approximation to unsteady aerodynamic phenomena. The following discussion applies to other similar pairs of parameters, such as $C_{L_{q}}$ and $C_{L_{\dot{\alpha}}}$, but the associated results are not reported in this paper because the dependencies identified for these parameters were less important for the overall aircraft dynamic motion than the dependencies for the pitching moment.

Model parameters $C_{m_{q}}$ and $C_{m_{\dot{\alpha}}}$ are not usually estimated separately and accurately from flight test data because conventional aircraft maneuvers performed for system identification 
analysis result in pitch rate and angle of attack rate time series that are nearly proportional to each other. These data are said to be highly correlated with each other, or nearly collinear. No parameter estimation method can correctly attribute the dependencies of the pitching moment coefficient on these two variables when they are highly correlated. Solutions to this problem include combining dependencies to estimate a single lumped derivative, using other analyses to provide prior information on some of the parameters, and redesigning the maneuver so that the pitch rate and angle of attack rate are less correlated. ${ }^{2,3}$ The approach taken for this paper was to redesign the maneuver.

Flight test data is sometimes used to verify the predictive capability of simulation models. Accurate knowledge of $C_{m_{q}}$ and $C_{m_{\dot{\alpha}}}$ from the flight test data is needed to fairly compare these estimates. Otherwise, discrepancies result and further effort is required to determine the source of the differences. Accurate knowledge of these parameters can also improve the modeling fidelity in flight regimes such as at high angles of attack, rapid maneuvering, take off, landing approaches, and loss of control scenarios.

This paper discusses accurate estimation of pitch rate and angle of attack rate derivatives from flight test data. The important characteristics of maneuvers that successfully decouple these dependencies are discussed, as well as some useful parameter estimation techniques. Flight test data from three different types of maneuvers are presented. The maneuvers are relatively simple to perform on most aircraft. Section II discusses the modeling and estimation problem in detail. Section III presents the experiment design and analysis method. The airplanes used to fly the maneuvers are described in Section IV. Flight test data and estimation results are presented and discussed in Section V.

\section{Problem Description}

The aerodynamic forces and moments acting on the aircraft can be modeled as truncated Taylor series expansions using the aircraft state and control variables for small perturbations from a reference flight condition. A model of the pitching moment coefficient is

$$
C_{m}=C_{m_{0}}+C_{m_{V}} \frac{\Delta V}{V_{0}}+C_{m_{\alpha}} \Delta \alpha+C_{m_{q}} \frac{q \bar{c}}{2 V}+C_{m_{\dot{\alpha}}} \frac{\dot{\alpha} \bar{c}}{2 V}+C_{m_{\delta_{e}}} \Delta \delta_{e}
$$

For convenience, $C_{m_{V}}$ here subsumes all terms that vary with airspeed. Inherent in Eq. (1) is the assumption that the flow is quasi-steady, and that aerodynamic forces and moments develop instantaneously from the aircraft motion. This type of model is in contrast to unsteady models, e.g. in Ref. 4, which depend upon the current and past history of the motion.

The pitch damping derivative $C_{m_{q}}$ quantifies the pitching moment generated by a pure pitch rate while all other variables are constant. For conventional aircraft configurations, it is mostly attributed to the additional lift on the horizontal tail due to rotation about the center of mass. Etkin ${ }^{5}$ derives the analytical expression for this effect as 


$$
C_{m_{q}}=-2 a_{t} V_{H} \frac{l_{t}}{\bar{c}}
$$

where $a_{t}$ is the lift-curve slope of the horizontal tail, $V_{H}$ is the horizontal tail volume, and $l_{t}$ is the distance between the center of mass and the aerodynamic center of the horizontal tail.

The angle of attack rate derivative $C_{m_{\dot{\alpha}}}$ quantifies the pitching moment resulting from a sudden change in angle of attack, or equivalently, a pure vertical acceleration, while all other variables are constant. An analytical expression for this parameter similar to Eq. (2) is less straightforward because it represents an approximation to unsteady aerodynamic phenomena, which are more complex in nature. As discussed by Etkin, ${ }^{5}$ the pitching moment increment arising from an angle of attack perturbation can be written as the aerodynamic transfer function

$$
\Delta C_{m}(s)=\left(C_{m_{\alpha}}+C_{m_{\dot{\alpha}}} s+C_{m_{\ddot{\alpha}}} s^{2}+\ldots\right) \Delta \alpha(s)
$$

The steady-state value $C_{m_{a}} \Delta a$ is reached after transients from higher-order unsteady aerodynamic terms have decayed. Truncating this infinite power series after the first two terms results in a quasi-steady model equivalent to Eq. (1).

Another way to view the angle of attack rate derivative is the "lag of the downwash" concept by Glauert and Cowley. ${ }^{6}$ Here it is assumed that the flow surrounding the horizontal tail is affected by the vorticity of the main wing following a transport delay $\tau=l_{t} / V$. The angle of attack increment at the tail is

$$
\Delta \alpha_{t}(t)=\Delta \alpha(t)-\Delta \alpha(t-\tau) \frac{\partial \epsilon}{\partial \alpha}
$$

assuming a linear downwash model. Truncating the power series expansion of $\Delta a(t-\tau)$ in terms of $\tau$ after the first two terms simplifies Eq. (4) to

$$
\Delta \alpha_{t}(t)=\left(1-\frac{\partial \epsilon}{\partial \alpha}\right) \Delta \alpha(t)+\frac{l_{t}}{V} \frac{\partial \epsilon}{\partial \alpha} \dot{\alpha}(t)
$$

The expression in Eq. (4) is unsteady because it depends on previous values of angle of attack, but the approximation in Eq. (5) is quasi-steady because it depends on instantaneous values. Lag of the downwash is an unsteady aerodynamic effect that is present in normal flight conditions, which is in contrast to frequency-dependent unsteady aerodynamics that are encountered at higher angles of attack. ${ }^{7}$ Using Eq. (5), the analytical expression for the angle of attack rate derivative is

$$
C_{m_{\dot{\alpha}}}=-2 a_{t} V_{H} \frac{l_{t}}{\bar{c}} \frac{\partial \epsilon}{\partial \alpha}
$$


While Eqs. (2) and (6) differ only by the downwash derivative, the physical origins from which pitch damping and the angle of attack rate derivative arise are different. In addition to unsteady aerodynamics, the angle of attack rate derivative can also confound effects due to aeroelasticity, compressibility, and changes in center of mass.

The accurate estimation of model parameters in Eq. (1) from flight test data is a common goal for system identification analyses. Conventional maneuvers performed to obtain flight test data involve small perturbations about a reference condition, e.g. using frequency sweep, multisine, or step-type inputs. For these types of maneuvers, pitch rate and angle of attack rate time series are almost identical to each other. As discussed in Ref. 2, this correlation can be understood from examining the differential equation for the angle of attack

$$
\begin{aligned}
& \dot{\alpha}=q-\frac{\bar{q} S}{m V \cos \beta} C_{L}+\frac{g(\cos \alpha \cos \phi \cos \theta+\sin \alpha \sin \theta)}{V \cos \beta}-\tan \beta(p \cos \alpha+r \sin \alpha) \\
& -\frac{X_{T} \sin \alpha}{m V \cos \beta}
\end{aligned}
$$

The terms on the right side of Eq. (7) are ordered in descending magnitude for typical maneuvers. The first term is the pitch rate, arising from kinematic terms in the equations of motion. The second and third terms are the contributions from aerodynamic lift and gravity forces. The last two terms arise from lateral kinematics and thrust forces. For small perturbation maneuvers, the kinematic pitch rate term dominates the angle of attack rate equation. It is therefore expected that $q$ and $\dot{\alpha}$ are almost equal and therefore highly correlated in these types of maneuvers.

To illustrate this correlation, flight test data for a typical small perturbation maneuver using the T-2 aircraft (described in Section IV) is shown in Fig. 1. Multisine inputs (described in Section III) were applied to the elevator, aileron, and rudder surfaces while the aircraft was in a straight and level flight condition to elicit small perturbation responses about all axes of the aircraft. The input bandwidth was 0.2 to $2.2 \mathrm{~Hz}$, which spanned the expected short period and Dutch roll frequencies. This maneuver produced good ranges and signal-to-noise ratios in the response variables for linear modeling. Angle of attack rate was computed by correcting wing-tip vane measurements to the center of mass and smoothly differentiating ${ }^{8}$ the data. A visual inspection of $q$ and $\dot{\alpha}$ in Fig. 1 shows they look similar. This notion is confirmed by the plot matrix in Fig. 2. The diagonal of this matrix labels the explanatory variables corresponding to the model parameters. The upper triangle consists of pairwise cross plots of the data. Units are not indicated because the shape of the data was examined rather than the magnitude. Most combinations of variables covered the space well and did not indicate a strong linear dependence. However, the pitch rate and angle of attack rate moved in proportion to each other and their cross plot resembles a thin straight line, which visually reflects their correlation.

Pairwise correlations for two series, $x_{j}$ and $x_{k}$, are defined as 


$$
r_{j k}=\frac{\sum_{i=1}^{N}\left(x_{j}(i)-\bar{x}_{j}\right)\left(x_{k}(i)-\bar{x}_{k}\right)}{\sqrt{\sum_{i=1}^{N}\left(x_{j}(i)-\bar{x}_{j}\right)^{2} \sqrt{\sum_{i=1}^{N}\left(x_{k}(i)-\bar{x}_{k}\right)^{2}}}}
$$

where $\bar{x}$ denotes the mean of regressor $x$. This metric ranges between \pm 1 and is a measure of linear dependence between the pair of measurements. Correlations with absolute value greater than 0.9 are considered highly correlated. ${ }^{8}$ The lower triangle in Fig. 2 indicates the pairwise correlation for the explanatory variables. Only the pair corresponding to $C_{m_{q}}$ and $C_{m_{\dot{\alpha}}}$ were highly correlated. Diagnostic tests 8,9 indicated that multicollinearity was not an issue, so that pairwise correlations were sufficient in diagnosing the collinearity.

Figure 3 shows time histories of the angle of attack rate and the first three terms on the right side of Eq. (7). The lift coefficient was computed as

$$
C_{L}=-C_{Z} \cos \alpha+C_{X} \sin \alpha
$$

where

$$
\begin{gathered}
C_{X}=\frac{m a_{x}}{\bar{q} S}-\frac{X_{T}}{\bar{q} S} \\
C_{Z}=\frac{m a_{z}}{\bar{q} S}
\end{gathered}
$$

Substituting measured data in these equations to compute the lift coefficient did not require models of the nondimensional aerodynamic coefficients. The kinematic pitch rate contribution was dominant and described $83 \%$ of the variation in the angle of attack rate. The contributions from the lift force were small, and the contribution from gravity was approximately a constant.

Correlated data is problematic for estimating model parameters because variation in the dependent variable cannot be accurately attributed to the explanatory variables. The resulting parameter estimates are biased and have increased uncertainties. One solution to this problem is to supply prior information to the estimator from a separate test or analysis using a Bayesian formulation. ${ }^{8}$ Care must be taken because any error in the supplied information diminishes the accuracy of the remaining estimated parameters. A second solution, which is routinely used, is to adjust the model structure so that the explanatory variables are not correlated. For the pitching moment coefficient, the pitch rate and angle of attack rate dependencies are combined into the one parameter, $C_{m_{q}}+C_{m_{\dot{\alpha}}}$ The total variation is then attributed to pitch rate, rather than angle of attack rate, for ease of implementation in statespace models. Attributing this variation instead to the angle of attack rate would result in an implicit equation in $\dot{\alpha}$ Lumping the dependencies together will bias parameter estimates and degrade predictive capability for flight regimes where pitch rate and angle of attack rate are no longer correlated. Furthermore, models based on numerical or analytical predictions 
cannot be reliably updated from flight test results because the parameters are confounded. A third solution, which is the approach used in this paper, is to redesign the maneuvers such that the data are less correlated.

Continuing the T-2 example, Fig. 4 shows the parameter estimation results from five repeated small-perturbation maneuvers, with pitch rate and angle of attack rate dependencies estimated separately and as a combined parameter. Results are shown using the equationerror method described in Section III, and error bars reflect the $2 \sigma$ uncertainty bound. The variation in airspeed was small, so $C_{m_{V}}$ was not estimated. In the first case, $C_{m_{\dot{\alpha}}}$ was not included in the model structure and $C_{m_{q}}$ subsumed both pitch rate and angle of attack rate dependencies. The parameter estimate scatter and uncertainty bounds were both small. In the second case, $C_{m_{\dot{\alpha}}}$ was included in the model structure. The estimates of $C_{m_{\alpha}}$ and $C_{m_{\delta_{e}}}$ were only slightly affected by whether or not $C_{m_{\dot{\alpha}}}$ was included in the model. However, the estimates of $C_{m_{q}}$ incurred a significant bias, and estimates of both $C_{m_{q}}$ and $C_{m_{\dot{\alpha}}}$ had large uncertainties and large amounts of scatter between maneuvers. Estimates of $C_{m_{\dot{\alpha}}}$ were statistically zero, or nearly so.

The nonlinearities present in Eq. (7) offer creative opportunities for designing maneuvers that reduce the amount of correlation between pitch rate and angle of attack rate. Maine and Iliff $^{2}$ investigated two maneuvers that used large bank angles to decorrelate pitch rate and angle of attack rate through the gravity term in Eq. (7). The first maneuver, called a smooth roll, was a steady $360 \mathrm{deg}$ roll performed between $30-60 \mathrm{deg} / \mathrm{s}$ while elevator pulses were applied. The second maneuver, called a two-point hesitation roll, involved applying an elevator pulse with wings level, then rolling the aircraft $180 \mathrm{deg}$ and repeating the elevator pulse while inverted. In both these maneuvers, $\cos \varphi$ varied between 1 and -1 , which appreciably lowered the correlation between pitch rate and angle of attack rate. Parameter estimation results were repeatable and in rough agreement with simple analytical predictions. It was noted that this maneuver was performed using a small and agile jet trainer, and that it may not be acceptable for other aircraft.

Jategaonkar and Gopalratnam ${ }^{3}$ presented an alternative maneuver that also exploited the gravity term in Eq. (7) but avoided rolling the aircraft into an inverted position. The maneuver involved applying multistep elevator inputs while in straight and level flight, and then also while banked to at least $40 \mathrm{deg}$. The throttle was also moved to vary airspeed. These changes were sufficient to reduce the correlation between $q$ and $\dot{\alpha}$ and estimated values of the model parameters were similar to wind tunnel predictions.

Any maneuver that sufficiently decorrelates $q$ and $\dot{\alpha}$ can be used for identification. Successful maneuvers in the past have relied upon achieving a large bank angle and using gravity to achieve the decorrelation. In this paper, other strategies and maneuvers were used to decorrelate the pitch rate and angle of attack rate. The maneuvers demonstrated are applicable for testing with most aircraft, and are relatively simple to implement. The only additional costs incurred to achieve this information are longer record lengths of data or 
additional maneuvers, and/or instrumenting the aircraft to use computerized control surface inputs.

\section{Methods}

This section briefly summarizes a few pertinent test and analysis techniques. Software for the input design, post-flight data compatibility analysis, signal processing, data analysis, and parameter estimation used here are available in a MATLAB ${ }^{\circledR}$ toolbox called System IDentification Programs for AirCraft, or SIDPAC, ${ }^{10}$ which is associated with Ref. 8.

\section{III.A. Orthogonal Phase-Optimized Multisine Excitation}

Some maneuvers discussed used orthogonal phase-optimized multisines, which are perturbation inputs developed at NASA Langley Research Center to achieve efficient excitation of multiple inputs for system identification. References 8, 11, and 12 provide details on the input design, and Ref. 13 presents numerous flight test examples.

Each perturbation input $\Delta u_{j}$, applied to input $u_{j}$, is a summation of sinusoids of the form

$$
\Delta u_{j}(t)=A_{j} \sum_{k \in K_{j}} a_{k} \sin \left(\frac{2 \pi k}{T} t+\phi_{k}\right)
$$

The selection of the maneuver duration $T$ determines the fundamental frequency of the excitation $1 / T$. Multiples $k$, chosen over a desired frequency bandwidth, then define the excitation frequencies $k / T$. Because harmonic sinusoids with different frequencies are orthogonal in both the time and frequency domains, the harmonics $k$ can be assigned to different inputs and applied simultaneously without correlating the inputs. The relative power at each frequency can be chosen arbitrarily using $a_{k}$. Once these parameters have been designed, the phase angles $\varphi_{k}$ are optimized to produce inputs with minimal relative peak factors, to keep the aircraft relatively close to the desired flight condition. The input is scaled using gain $A_{j}$ according to desired response levels and signal-to-noise ratios.

\section{III.B. Equation Error Parameter Estimation}

Given adequate data, many different methods of parameter estimation can accurately identify the model parameters in Eq. (1). For simplicity, equation error and time-domain data were used in this work. The method is briefly introduced here, and more details can be found in Ref. 8. Equation error is often reduced to an ordinary least squares problem, i.e.

$$
\mathbf{z}=\mathbf{X} \boldsymbol{\theta}+\mathbf{v}
$$

where $\mathbf{z}$ is the dependent variable, $\mathbf{X}$ is a matrix of regressors or explanatory variables, $\boldsymbol{\theta}$ is a vector of unknown constants to be estimated, and $\mathbf{v}$ is a vector of equation errors. The cost function

$$
J(\boldsymbol{\theta})=\frac{1}{2}(\mathbf{z}-\mathbf{X} \boldsymbol{\theta})^{T}(\mathbf{z}-\mathbf{X} \boldsymbol{\theta})
$$


is minimized by the optimal solution for the model parameters

$$
\hat{\boldsymbol{\theta}}=\left(\mathbf{X}^{T} \mathbf{X}\right)^{-1} \mathbf{X}^{T} \mathbf{z}
$$

Equation (1) can be rearranged into the canonical form of Eq. (13) for estimating the model parameters. The dependent variable is the nondimensional pitching moment coefficient, which can be computed from measured data as

$$
C_{m}=\frac{1}{\bar{q} S \bar{c}}\left[I_{y y} \dot{q}+\left(I_{x x}-I_{z z}\right) p r+I_{x z}\left(p^{2}-r^{2}\right)-I_{e} \Omega_{e} r-M_{T}\right]
$$

The last two terms account for rotational momenta and thrust moments from rotating engines. The pitch acceleration $\dot{q}$ was numerically computed from angular rate data using a smoothed differentiation technique. ${ }^{8}$ Equation (16) accounts for the full nonlinear dynamics of the aircraft motion, as well as time-varying dynamic pressure and mass properties. The regressor matrix and model parameter vector are

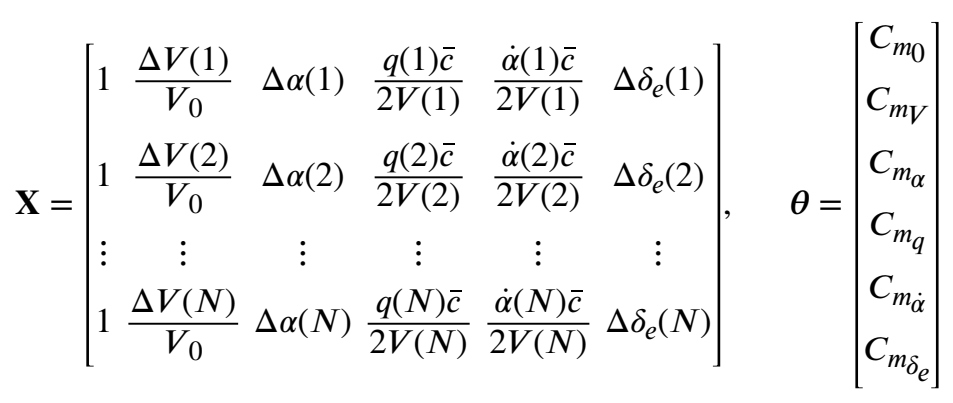

For the T-2 flight test data in straight and level flight, the bottom plot in Fig. 1 shows the calculated pitching moment and the model output computed using equation error. The match was very close and had coefficient of determination $R^{2}=0.95$, indicating a good fit to the data.

Etkin $^{5}$ discusses normalizing the rate variables by $\bar{c} / 2 V_{0}$ in the small disturbance case, and by $\bar{c} / 2 V$ in the general case. Using the reference airspeed $V_{0}$ in the normalization can reduce correlation of the other explanatory variables with airspeed for small perturbation maneuvers. However, as shown in Fig. 2, correlations with airspeed are among the lowest in small perturbation maneuvers. Because some of the maneuvers discussed involve appreciable changes in airspeed, $\bar{c} / 2 V$ was used for normalization. No noticeable differences were observed in the results for the small-perturbation maneuvers.

Ordinary least squares theory assumes that the regressor matrix $\mathbf{X}$ is measured without error. For this reason, an effort was made to improve the data prior to estimation. A data compatibility analysis ${ }^{8}$ was employed to remove time skew, bias, and scale factor errors in the measurements. The regressors were also smoothed to remove measurement noise, which would bias parameter estimates.

The uncertainty in the parameter estimates is 8,14 


$$
\operatorname{cov}(\hat{\boldsymbol{\theta}})=\left(\mathbf{X}^{T} \mathbf{X}\right)^{-1} \mathbf{X}^{T} E\left[\mathbf{v v} \mathbf{v}^{T}\right] \mathbf{X}\left(\mathbf{X}^{T} \mathbf{X}\right)^{-1}
$$

where the sample autocorrelation function is used to estimate the covariance of the equation error from the residuals. The square root of the diagonal terms in this matrix are the standard errors of the parameter estimates. Equation (18) is more complex than that typically used in ordinary least squares, and arises from serial correlations in the data due to model structure error, which are not assumed by the theory.

Sometimes there is not enough information content in a single maneuver to estimate all the model parameters of interest. When using equation error, data from multiple maneuvers can be combined easily, and in multiple ways, ${ }^{8}$ to increase the data information content. One simple procedure is to concatenate the data from the maneuvers. For $m$ maneuvers, Eq. (13) is augmented to become

$$
\left[\begin{array}{c}
\mathbf{z}_{1} \\
\mathbf{z}_{2} \\
\vdots \\
\mathbf{z}_{m}
\end{array}\right]=\left[\begin{array}{c}
\mathbf{X}_{1} \\
\mathbf{X}_{2} \\
\vdots \\
\mathbf{X}_{m}
\end{array}\right] \boldsymbol{\theta}+\left[\begin{array}{c}
\mathbf{v}_{1} \\
\mathbf{v}_{2} \\
\vdots \\
\mathbf{v}_{m}
\end{array}\right]
$$

and the analysis then proceeds in the usual fashion. Combining data from multiple maneuvers is one way to account for data information content deficiencies. This technique assumes that the model structure is the same, and that the error variances are similar, for all maneuvers. When the error variances differ among the maneuvers, a weighted average results. This could be the case when different types of maneuvers are combined, because the error variance includes model structure error, which is maneuver dependent.

\section{Aircraft and Flight Tests}

The T-2 and Bat-4 aircraft were used to fly the maneuvers. These airplanes are pictured in Fig. 5 with mass and geometry properties given in Table 1. Both aircraft were tested using the NASA Langley AirSTAR (Airborne Subscale Transport Aircraft Research) flight test facility, where a research pilot flew the aircraft remotely using synthetic vision drawn from telemetry data and a map of the local terrain. Multisine inputs were applied by the pilot pushing and holding a button on the thrust lever while flying the airplane. Data were telemetered at $200 \mathrm{~Hz}$, and downsampled to $50 \mathrm{~Hz}$ for real-time modeling. For more information on AirSTAR, see Ref. 15.

The T-2 aircraft is a 5.5\% dynamically-scaled model of a generic, commercial, jet transport aircraft. It has twin jet engines mounted under the wings and retractable tricycle landing gear. The airplane was equipped with a micro Inertial Navigation System (INS), which provided three-axis translational accelerometer measurements, angular rate measurements, estimated attitude angles, and Global Positioning System (GPS) velocity and position. An inertial measurement unit (IMU) gave additional accelerometer and gyroscope measurements with lower latency. Air data probes attached to booms mounted on each wingtip measured angle of attack, flank angle, static pressure, and dynamic pressure. 
Measurements from static pressure sensors and ambient temperature sensors were used to compute air density and altitude. Engine speed was measured using a tachometer and used as input to an engine model to compute thrust. The engine model was identified from ground test data with adjustments for ram drag identified from flight data. Potentiometers on the rotation axes of the control surfaces were used to measure control surface deflections. Mass properties were computed based on measured fuel flow, pre-flight weight and balance, and inertia measurements done on the ground for the aircraft without fuel. The T-2 aircraft has 16 separate control surfaces. For the flight data analyzed in this work, only the elevator, aileron, and rudder control surfaces were deflected.

The Bat- 4 is a fixed-wing, remotely-piloted, unmanned aircraft. It has fixed tricycle landing gear, a twin-boom tail section, a single fixed-pitch pusher propeller, and a $6.6 \mathrm{in}^{3}$ two-stroke gasoline engine. It is manufactured by the MLB Company and marketed as an aerial mapping, surveillance, and payload platform. The airplane was fitted with custom hardware and sensors for research flight testing. Onboard sensors included rate gyros, translational accelerometers, outside air temperature sensors, GPS, and estimated Euler angles. Vanes on dual wing-tip mounted air-data booms provided angle of attack and flank angle measurements. Pressure taps on the booms also measured static and total pressures, from which airspeed and altitude were computed. Fuel flow measurements were combined with a computer-aided design (CAD) model of the aircraft and pre-flight weight and balance data to model the changing mass properties during flight. The ailerons were moved asymmetrically to apply aerodynamic roll moments, and the ruddervators were moved both symmetrically and asymmetrically to apply aerodynamic pitch and yaw moments, respectively.

\section{Results and Discussion}

\section{V.A. q-Isolation Maneuvers}

Traditional pullup or pushover maneuvers involve the pilot slowly pulling back or pushing forward on the stick. Similar maneuvers were flown to separate pitch rate and angle of attack rate, with the important difference that angle of attack was held approximately constant during the maneuver. This was done to create a zero angle of attack rate and a non-zero pitch rate, consistent with the definition of the $C_{m_{q}}$ parameter.

These $q$-isolation maneuvers were developed using the AirSTAR piloted flight simulator, and were refined and practiced during simulation sessions prior to flight test. The most timeconsuming part of the maneuver development was determining the initial conditions for the entry into the maneuver and for the start of the constant angle of attack phase. Once the maneuvers were developed and refined in the simulator, the two flight test maneuvers were completed successfully, with one repeat each, and provided excellent data for modeling. Having an angle of attack indicator at the pilot station and the use of an angle of attack command flight control law made the piloting task relatively easy. It is believed that the piloting task would also be manageable with other flight control laws, including direct stickto-surface control in un-augmented aircraft, as long as the pilot has a real-time display of angle of attack at the pilot station. 
For the pullup maneuver, the airplane was initially trimmed in level flight at an indicated airspeed of 70 knots. Power was set to 50\%, the nose was smoothly pushed over to a target pitch attitude of $-20 \mathrm{deg}$, and then back stick was applied to capture 6.0 deg angle of attack. This angle of attack was held as the airplane slowly pitched up, and recovery was initiated at a pitch attitude of $25 \mathrm{deg}$.

For the pushover maneuver, the airplane was initially trimmed in level flight at an indicated airspeed of 90 knots. Power was set to $66 \%$, the nose was smoothly pulled up to a target pitch attitude of $25 \mathrm{deg}$ nose up, and then the stick was relaxed to capture $4.5 \mathrm{deg}$ angle of attack. This angle of attack was held as the airplane slowly pitched down, and recovery was initiated at a pitch attitude of $-20 \mathrm{deg}$.

Figure 6 shows flight test data analyzed for a $q$-isolation pullup and a $q$-isolation pushover maneuver. Time $t=0$ corresponds to when the target angle of attack was captured. These plots show that a pitch rate registered while the other quantities were held approximately constant or varied slowly. The contributions to the angle of attack rate are shown in Fig. 7. The total value is almost zero throughout the maneuver because the angle of attack was mostly constant. The other contributions cancel out when summed together, and the kinematic pitch rate was less dominant than in the small-perturbation maneuver.

By design, pitch rate and angle of attack rate had a low level of correlation during this maneuver. This is confirmed by the cross plots in Fig. 8 and the low pairwise correlations between $C_{m_{q}}$ and $C_{m_{\dot{\alpha}}}$ of 0.15 and 0.10 . However, these plots also indicate that the airspeed, pitch rate, and elevator were correlated because all of those quantities changed slowly and in a similar manner. These $q$-isolation maneuvers could therefore not be used to independently estimate all the model parameters in Eq. (1).

However, because the correlations in the $q$-isolation maneuvers are complementary to those in the straight and level maneuvers, the two maneuvers were combined in the estimation analysis to achieve independent estimates of all the model parameters. The $q$-isolation maneuvers can therefore be used to augment information from conventional smallperturbation maneuvers and accurately separate effects from pitch rate and angle of attack rate. The straight and level maneuvers provided information on the angle of attack, elevator, and combined pitch rate and angle of attack rate. The $q$-isolation maneuvers provided information on the pitch rate, with angle of attack rate held approximately constant. Between the two maneuvers, there is enough independent information to estimate all the model parameters.

Figure 9 shows the estimated model parameters and associated $2 \sigma$ bounds. The first two maneuvers are $q$-isolation pullups and the last two are $q$-isolation pushovers. Each result was obtained using one $q$-isolation maneuver and five straight and level maneuvers. Fewer straight and level maneuvers could have been used, but this was simple to implement and improved the parameter uncertainties without significantly altering the parameter estimates. Estimates using all nine maneuvers together are given in Table 2, and are similar to those illustrated. The identified parameters show good consistency between maneuvers, and relatively small error bounds for most parameters. Scatter in the $C_{m_{V}}$ estimate is because this 
term subsumes the quantity $C_{m_{V}}+2 C_{m_{0}}$, and $C_{m_{0}}$ is different between maneuvers. Values of -53.8 and $-10.7 \mathrm{rad}^{-1}$ were expected for the pitch damping and angle of attack rate derivative from evaluating Eqs. (2) and (6). These values are similar to and in statistical agreement with the identified parameters. Wind tunnel testing using a similar aircraft model reported that unsteady aerodynamics for this type of aircraft at low angles of attack are negligible. ${ }^{16}$ Therefore, $C_{m_{\dot{\alpha}}}$ should be attributed to lag of the downwash, which corroborates these estimation results.

\section{V.B. Multiple-Mode Excitation}

The response of the classical airplane phugoid mode is primarily characterized by a slow oscillation in airspeed, pitch rate, and pitch angle at approximately constant angle of attack. Exciting this modal response in flight decorrelates pitch rate from angle of attack rate because the angle of attack remains relatively constant, similar to the $q$-isolation maneuver. For estimating both pitch rate and angle of attack rate dependencies, however, this type of maneuver would normally have to be combined with another maneuver, similar to the $q$ isolation maneuvers, to gain information for estimating all the model parameters. This can be done by also exciting the short period mode, which is characterized by oscillations in angle of attack and pitch rate at constant airspeed. Flight data containing responses from both these modes exhibit lower correlation between $q$ and $\dot{\alpha}$ because of the different modal responses. This type of data information can be achieved using multisine inputs.

Multisine inputs were designed for the Bat-4 to move the ruddervator and aileron surfaces in different ways. The inputs had a $40 \mathrm{~s}$ duration, uniform power spectra, and contained frequencies between 0.05 and $1.5 \mathrm{~Hz}$, which excited both phugoid and short period responses. These inputs were applied after the aircraft had been trimmed in straight and level flight. The longer maneuver duration and lower excitation frequencies were possible because this was the first flight test campaign in which the AirSTAR ground control station was used to fly an unmanned vehicle beyond visual range of the safety pilot. ${ }^{17}$

Flight test data for this maneuver are shown in Fig. 10. The elevator is considered the symmetric motion of the two ruddervator surfaces. The lower frequencies of the multisine input excited the phugoid mode, which is primarily evident in the airspeed and pitch angle measurements, and the higher frequencies excited the short period mode, which is apparent in the angle of attack and pitch rate measurements. The inputs created small perturbations from the reference condition.

Figure 11 shows the regressor cross plots and pairwise correlations. The experiment design resulted in enough phugoid motion so that pitch rate and angle of attack rate were decorrelated to a workable extent, indicated by a pairwise correlation coefficient of 0.56 . All other correlations were low to moderate, and the explanatory variables covered good ranges for modeling. Diagnostic metrics did not indicate a concern for multicollinearity.

In Fig. 12, the angle of attack rate is decomposed into its component parts. Although the kinematic pitch rate was still the most dominant term, changes in the lift and gravity terms were large enough to lower the correlation between pitch rate and angle of attack rate for 
accurate parameter estimation. The gravitational contribution changed because the airspeed and pitch angle, in addition to the other variables, varied. Likewise, the lift contribution changed because the lift force and airspeed varied. Although only about two cycles of the phugoid mode were evident in the maneuver, this duration was long enough to sufficiently reduce the regressor correlations.

Parameter estimates are shown in Fig. 13. In the first three maneuvers, each aileron and each ruddervator control surface was moved independently. In the fourth maneuver, the control surfaces were moved in the traditional way. Different maximum amplitudes on the deflections were applied in each maneuver. The parameter estimates are in statistical agreement among the four maneuvers. Based on Eq. (2), $C_{m_{q}}$ was expected to be about -11.8 $\operatorname{rad}^{-1}$. This approximation is questionable because of the unusual airplane design, but is in rough agreement with the results shown in Fig. 13. The estimates for $C_{m_{\dot{\alpha}}}$ were positive, which is not consistent with the lag of the downwash concept. The physical mechanism for this result is currently not understood, but interactions resulting from the pusher propeller and tail being in close proximity are suspected. Regardless of the parameter estimates for this specific airplane, the maneuver resulted in flight test data that had low correlations and good quality for modeling. Parameter estimates using all four maneuvers together are given in Table 2.

This type of maneuver is similar to conventional small-perturbation maneuvers used for system identification. The difference here is that the multisine excitation was designed to excite lower frequencies near the phugoid mode. These lower frequencies required longer records of data, to observe the slower response, and consequently a larger range in which to fly the airplane.

\section{V.C. Excitation Through Turns}

Maine and Iliff ${ }^{2}$ used a 360 deg roll to separate pitch rate and angle of attack rate. Jategaonkar and Gopalratnam ${ }^{3}$ also used this strategy, but reduced the bank angle to $40 \mathrm{deg}$ and added a segment of data in straight and level flight. Similar information can be extracted from data obtained from flight through a turn. Before AirSTAR was equipped with beyond visual range capability, operation in line of sight of the safety pilot restricted the aircraft to low maximum altitudes and ranges. Flight paths therefore often consisted of a short straight section followed by a 180 deg turn.

In the maneuvers that follow, multisine inputs were applied to the T-2 aircraft either continuously or intermittently as the aircraft flew a trajectory similar to a typical oval-shaped holding pattern. Flight data for one maneuver is shown in Fig. 14. The first $15 \mathrm{~s}$ consist of flying a straightaway with multisine excitation. The next $25 \mathrm{~s}$ of data were in a 45 deg bank angle turn, with the multisine still running. Normal operational limits restricted the bank angle from increasing much further. To maintain altitude, the pilot pulled back on the stick and increased angle of attack.

The correlation plots in Fig. 15 show that pitch rate and angle of attack rate had a correlation of 0.64 , which was sufficiently low for accurate parameter estimation. All other correlations 
were also low to moderate, and there was no indication of multicollinearity in the data.

Figure 16 shows that pitch rate and angle of attack rate became most different between 15 and $40 \mathrm{~s}$, when the aircraft was banked to $45 \mathrm{deg}$ and the gravitational contribution to angle of attack rate changed the most. Although this maneuver was sufficient for modeling, larger bank angles would have further decorrelated these quantities.

Parameter estimates are shown in Fig. 17. The first two maneuvers are continuous multisine excitation through the turns. These two maneuvers were flown in moderate turbulence, which degraded the estimation results. The second two maneuvers are intermittent excitation through the turns, and with light turbulence. Parameter estimates vary somewhat between maneuvers, particularly $C_{m_{V}}$, because the angle of attack ranged between 2 to $16 \mathrm{deg}$. This

large range in angle of attack includes known nonlinear phenomena such as stall and a noseslice departure, and abuses the linear model structure assumed in Eq. (1). However, estimates are similar to those shown in Fig. 9, and are in statistical agreement with analytical predictions.

This maneuver consisted of including the excitations while flying a standard racetrack loop, and was therefore simple to implement using the multisine inputs. Good modeling results were attained without further augmentation of the maneuver. Results could be further improved by achieving higher bank angles and limiting the variation in angle of attack through the turn.

\section{Concluding Remarks}

This paper discussed maneuvers suitable for separately quantifying the effects of pitch rate and angle of attack rate on the nondimensional aerodynamic pitching moment coefficient. Three types of maneuvers were presented. The first type was a $q$-isolation pullup or pushover maneuver flown at constant angle of attack. These maneuvers had nonzero pitch rate but approximately zero angle of attack rate. When combined with other maneuvers flown in straight and level flight, excellent modeling results were obtained. The second maneuver type involved exciting both the phugoid and short period modes using an optimized multisine input. The low-frequency excitation resulted in an airspeed variation that contributed towards decoupling pitch rate and angle of attack rate. The higher frequency excitations provided the remainder of the data information content necessary for estimating all model parameters. The last maneuver type applied optimized multisine excitations in both straight and level flight and in turning flight. The 45 deg bank angle was sufficient in decorrelating the data for modeling.

The maneuvers presented were relatively easy, if not routine in some instances, to accomplish. They can be performed with almost any aircraft instrumented for applying stored excitation inputs on the control surface commands, and did not require achieving large attitude angles. The maneuvers discussed are only a few of the possible maneuvers that can be used to decorrelate the data. Equation (7) suggests several possibilities for flying maneuvers that decorrelate the data. 
The $q$-isolation pullup and pushover maneuvers can be used to augment traditional smallperturbation maneuvers with information needed to accurately separate $C_{m_{q}}$ and $C_{m_{\dot{\alpha}}}$ These maneuvers did not need multisine inputs, and were relatively short maneuvers. If an aircraft has the capability for multisine inputs, these can be designed to excite the short period and phugoid mode together to lower correlations between $q$ and $\dot{\alpha}$ If the additional time and space needed to execute this maneuver is not available, multisine inputs that excite the short period mode can be continuously applied through straight and turning sections of a loop to achieve the necessary information.

The problem addressed here is a data information content deficiency; it is not a problem with parameter estimation per se. Past work has relied on the using the output-error method for parameter estimation. This work used the equation-error method, which is simple, fast, and provides a range of statistical metrics to the analyst. It also provides a simple means for combining maneuvers in the analysis, which was useful for the $q$-isolation maneuvers.

The new maneuvers presented, combined with the suggested parameter estimation techniques, represent a way to accurately separate pitching moment dependencies on pitch rate and angle of attack rate in an efficient manner. This information can be used to more precisely compare flight test results with other results, such as from simple analytical predictions, numerical aerodynamic codes, and wind tunnel tests. These maneuvers can also aide in building realistic flight simulation databases for unusual flight conditions where pitch rate and angle of attack rate affect the aircraft motion in different ways.

\section{Acknowledgments}

This work was funded under the NASA Aviation Safety Program, Integrated Resilient Aircraft Control and Vehicle Safety Systems Technology project, as well as the NASA Aeronautics Research Mission Directorate (ARMD) Learn-To-Fly project. The efforts of the AirSTAR flight test team at NASA Langley Research Center in modifying and testing the aircraft and associated systems, calibrating the instrumentation, and carrying out the flight operations are gratefully acknowledged.

\section{Nomenclature}

$\begin{array}{ll}\boldsymbol{a}_{\boldsymbol{x}}, \boldsymbol{a}_{z} & \text { body-axis translational accelerations, } \mathrm{g} \\ \boldsymbol{C}_{\boldsymbol{l}} & \text { lift coefficient } \\ \boldsymbol{C}_{\boldsymbol{m}} & \text { pitching moment coefficient } \\ \boldsymbol{C}_{\boldsymbol{X}}, \boldsymbol{C}_{\boldsymbol{Z}} & \text { body-axis force coefficients } \\ \bar{c} & \text { mean aerodynamic chord, } \mathrm{ft} \\ \operatorname{cov}(.) & \text { covariance } \\ \boldsymbol{E}[.] & \text { expectation operator } \\ \boldsymbol{g} & \text { gravitational acceleration, } \mathrm{ft} / \mathrm{s}^{2} \\ \boldsymbol{I} \cdot \boldsymbol{} & \text { inertia components, slug- } \mathrm{ft}^{2}\end{array}$




\begin{tabular}{|c|c|}
\hline$I_{e}$ & engine rotor inertia, slug- $\mathrm{ft}^{2}$ \\
\hline$M_{T}$ & thrust pitching moment, ft-lbf \\
\hline$m$ & mass, slug \\
\hline$N$ & number of data points \\
\hline$p, q, r$ & body-axis angular rates, $\mathrm{rad} / \mathrm{s}$ \\
\hline $\bar{q}$ & dynamic pressure, $\mathrm{lbf} / \mathrm{ft}^{2}$ \\
\hline$R^{2}$ & coefficient of determination \\
\hline$S$ & wing reference area, $\mathrm{ft}^{2}$ \\
\hline$s$ & Laplace variable \\
\hline$t$ & time, $\mathrm{s}$ \\
\hline $\boldsymbol{V}$ & true airspeed, $\mathrm{ft} / \mathrm{s}$ \\
\hline$X_{T}$ & body-axis thrust force, $\mathrm{lbf}$ \\
\hline$x, y, z$ & body axes \\
\hline$a$ & angle of attack, rad \\
\hline$\beta$ & angle of sideslip, rad \\
\hline$\Delta$ & perturbation from trim \\
\hline$\delta_{e}$ & elevator deflection, rad \\
\hline $\boldsymbol{\varepsilon}$ & downwash angle, rad \\
\hline$\sigma$ & standard deviation \\
\hline$\varphi, \theta, \psi$ & Euler angles, rad \\
\hline$\Omega$ & engine speed, $\mathrm{rad} / \mathrm{s}$ \\
\hline $\mathbf{0}$ & reference value \\
\hline $\mathrm{cm}$ & center of mass \\
\hline-1 & inverse \\
\hline$T$ & transpose \\
\hline$\cdot$ & time derivative \\
\hline$\hat{~}$ & estimate \\
\hline
\end{tabular}

\section{References}

1. Bryan G, Stability in Aviation, Macmillian and Co., 1911. 
2. Maine R. and Iliff K, "Maximum Likelihood Estimation of Translational Acceleration Derivatives from Flight Data," Journal of Aircraft, Vol. 16, No. 10, 10 1979, pp. 674-679.

3. Jategaonkar R. and Gopalratnam G, "Two Complementary Approaches to Estimate Downwash Lag Effects from Data," Journal of Aircraft, Vol. 28, No. 8, 8 1991, pp. 540-542.

4. Klein V. and Noderer K, "Modeling of Aircraft Unsteady Aerodynamic Characteristics: Part 1 Postulated Models," Tech. Rep. TM-109120, NASA, Hampton, VA, 51994.

5. Etkin B, Dynamics of Flight, John Wiley \& Sons, 1959.

6. Glauert H. and Cowley W, "The Effect of the Lag of the Downwash on the Longitudinal Stability of an Airplane," Tech. Rep. Reports and Memoranda No. 718, British Aeronautical Research Council, 21921.

7. Klein V, Murphy P, Curry T, and Brandon J, “Analysis of Wind Tunnel Longitudinal Static and Oscillatory Data of the F-16XL Aircraft,” Tech. Rep. TM-97-206276, NASA, Hampton, VA, 12 1997.

8. Morelli E. and Klein V, Aircraft System Identification:Theory and Practice, 2nd edition, Sunflyte Enterprises, Williamsburg, VA, 2016.

9. Belsley D, Kuh E, and Welsch R, Regression Diagnostics, Wiley Series in Probability and Mathematical Statistics, Wiley, 1980.

10. Morelli E, "System IDentification Programs for AirCraft (SIDPAC)," http://software.nasa.gov, Accessed: 2016-12-06.

11. Morelli E, "Multiple Input Design for Real-Time Parameter Estimation in the Frequency Domain," No. REG-360 in 13th IFAC Conference on System Identification, IFAC, Rotterdam, The Netherlands, August 2003.

12. Morelli E, "Flight-Test Experiment Design for Characterizing Stability and Control of Hypersonic Vehicles," Journal of Guidance, Control, and Dynamics, Vol. 32, No. 3, May-Jun 2009, pp. 949 959.

13. Morelli E, "Flight Test Maneuvers for Efficient Aerodynamic Modeling," Journal of Aircraft, Vol. 49, No. 6, Nov-Dec 2012, pp. 1857-1867.

14. Morelli E. and Klein V, "Accuracy of Aerodynamic Model Parameters Estimated from Fligh Test Data," Journal of Guidance, Control, and Dynamics, Vol. 20, No. 1, Jan-Feb 1997, pp. 74-80.

15. Cunningham K, Foster J, Morelli E, and Murch A, "Practical Application of a Subscale Transport Aircraft for Flight Research in Control Upset and Failure Conditions," No. 2008-6200 in Atmospheric Flight Mechanics Conference, AIAA, Honolulu, HI, August 2008.

16. Murphy P. and Klein V, "Transport Aircraft System Identification from Wind Tunnel Data," No. 2008-6202 in Atmospheric Flight Mechanics Conference, AIAA, Honolulu, HI, August 2008.

17. Cunningham K, Cox D, Riddick S, and Laughter S, “AirSTAR Beyond Visual Range System Description and Preliminary Test Results," No. 2016-0882 in Guidance, Navigation, and Control Conference, AIAA, San Diego, CA, January 2016. 

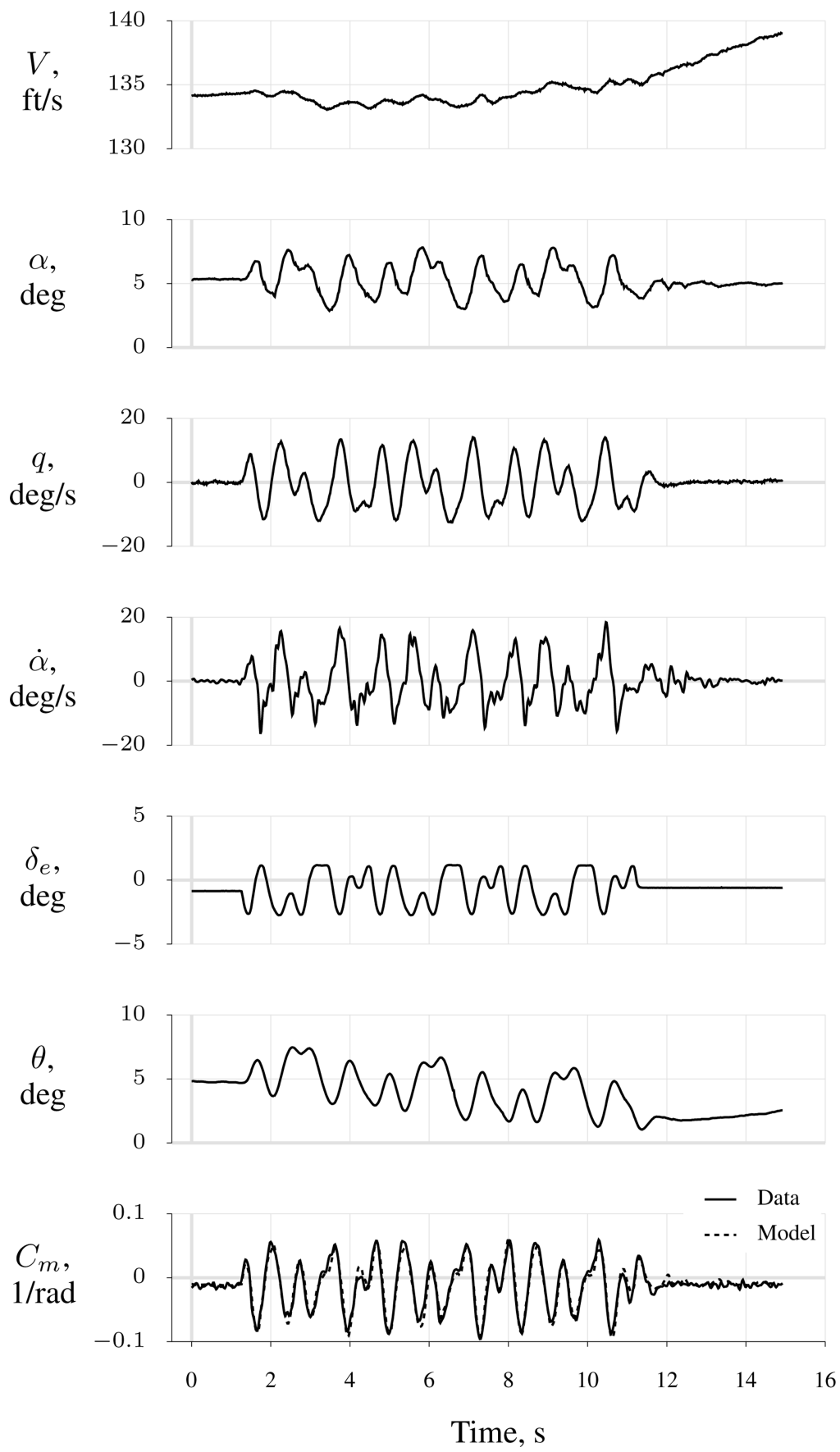

Figure 1.

Measurement time histories, T-2 straight and level maneuver 


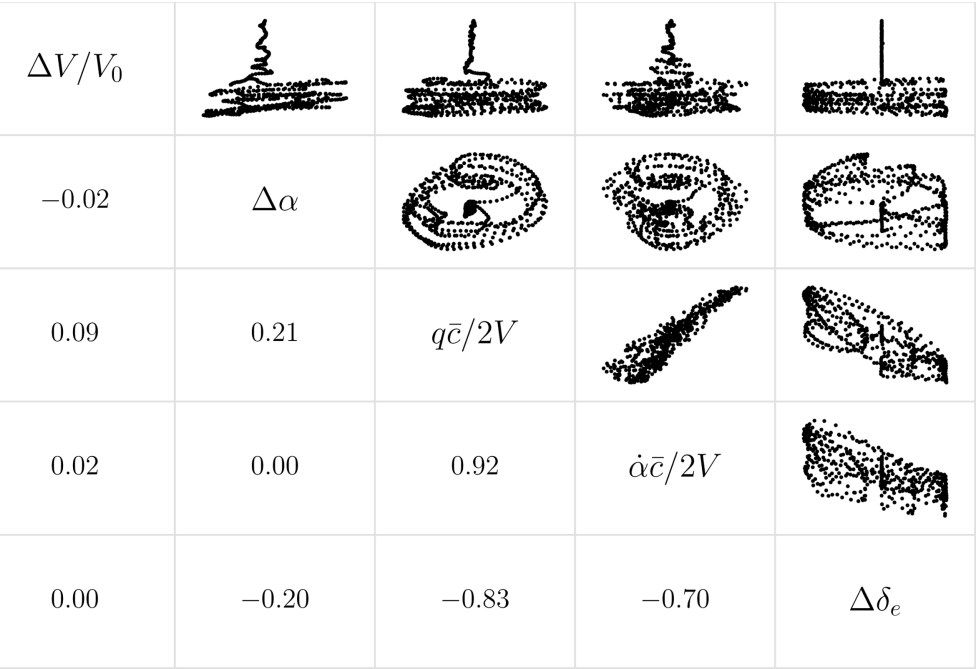

Figure 2.

Regressor cross plots and pairwise correlations, T-2 straight and level maneuver 


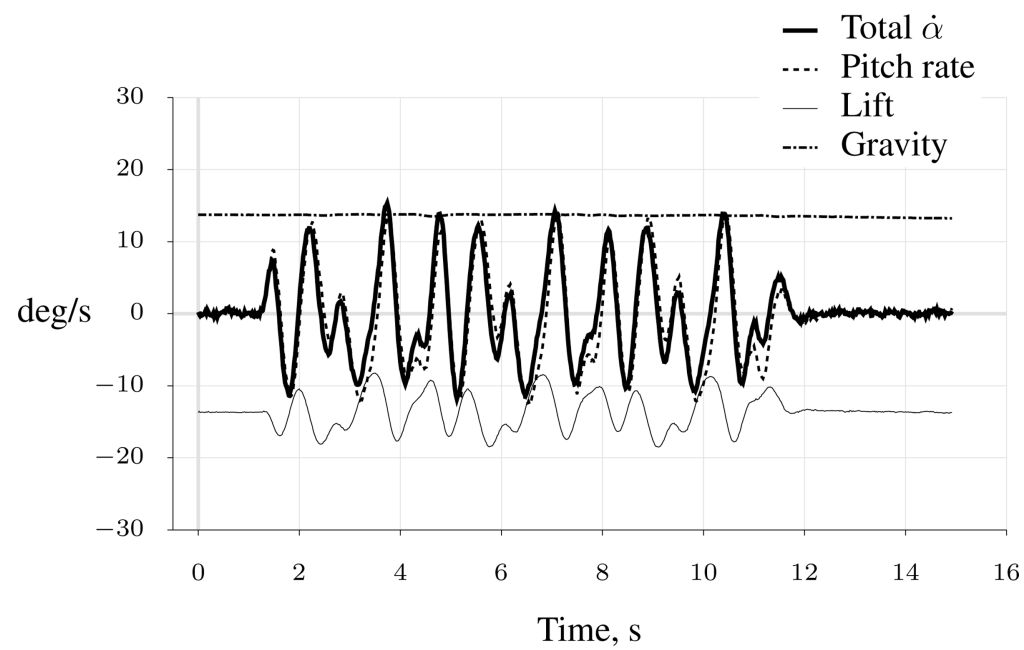

Figure 3.

Angle of attack rate contribution time histories, T-2 straight and level maneuver 

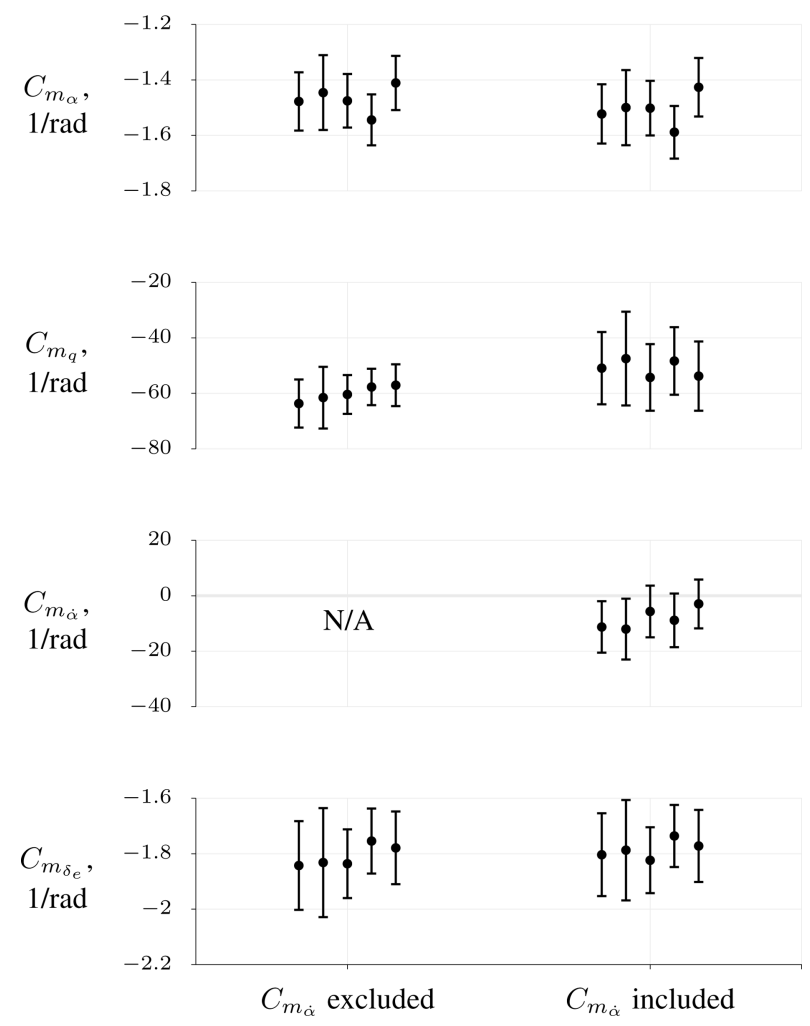

Figure 4.

Parameter estimates, T-2 straight and level maneuvers 


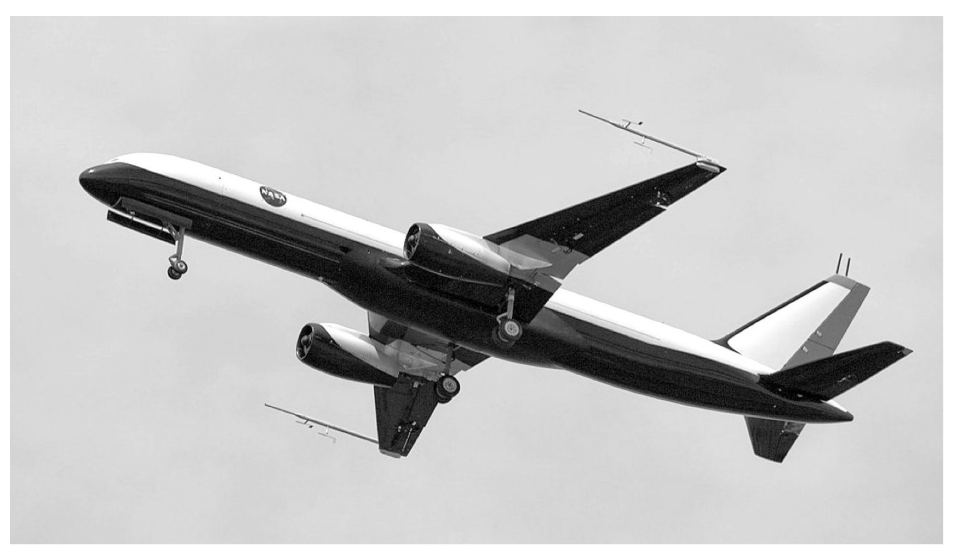

(a) T-2 airplane (credit: NASA Langley Research Center)

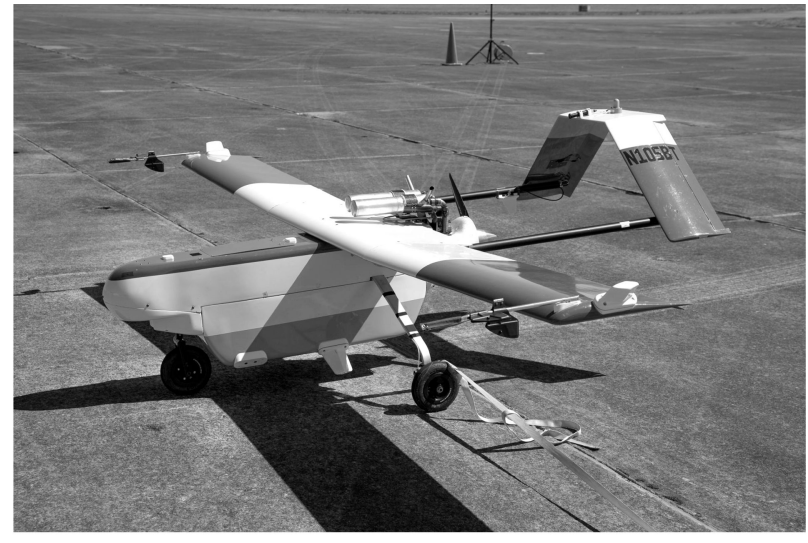

(b) Bat-4 airplane (credit: NASA Wallops Flight Facility)

Figure 5.

Test aircraft 

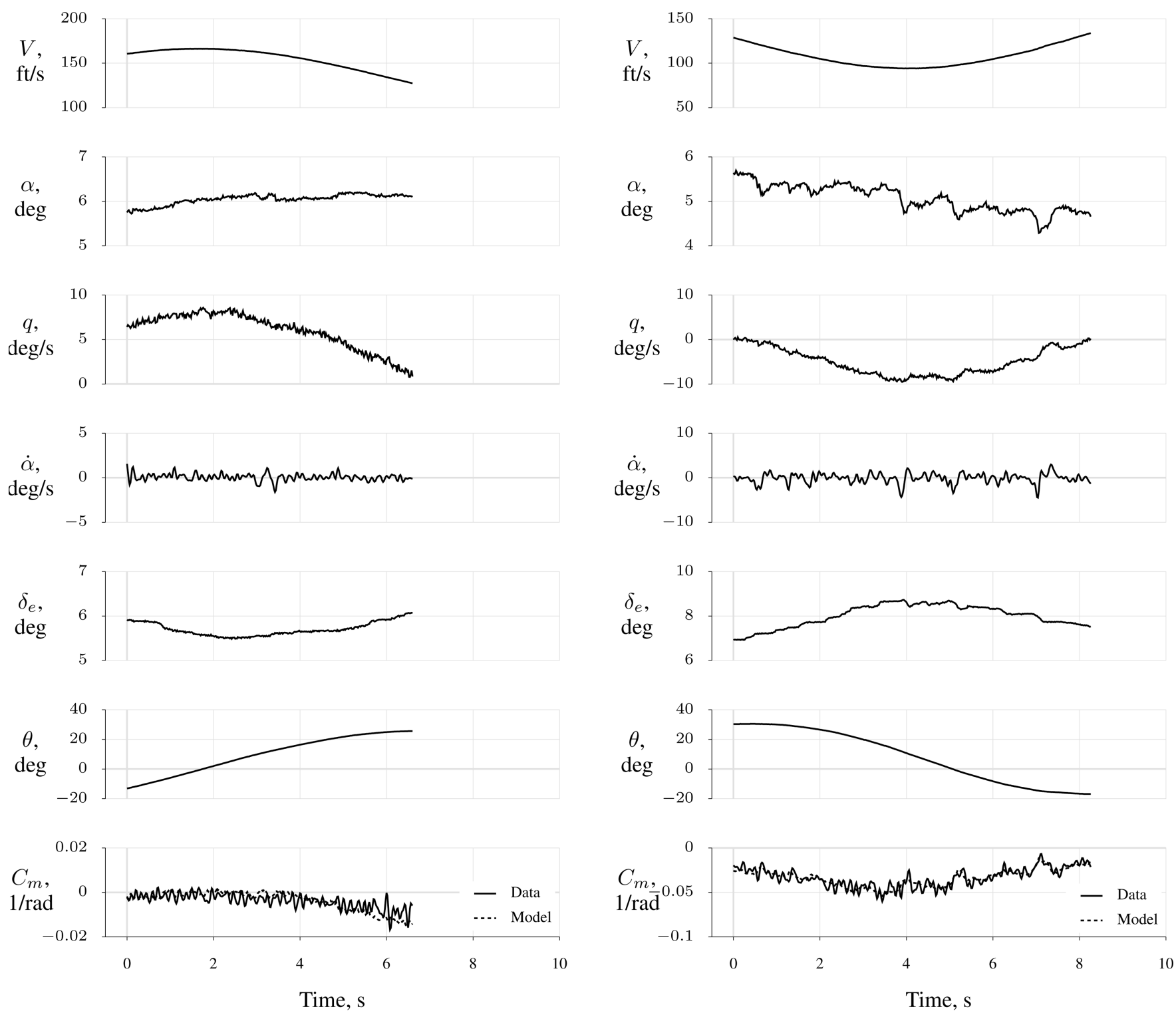

(a) Pullup maneuver

(b) Pushover maneuver

Figure 6.

Measurement time histories, T-2 $q$-isolation maneuvers 
Grauer et al.

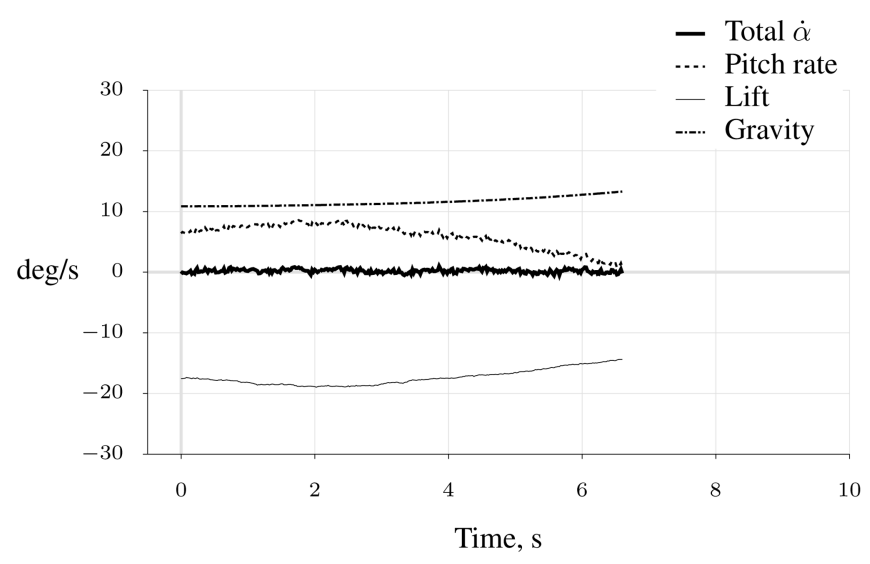

(a) Pullup maneuver
Page 24

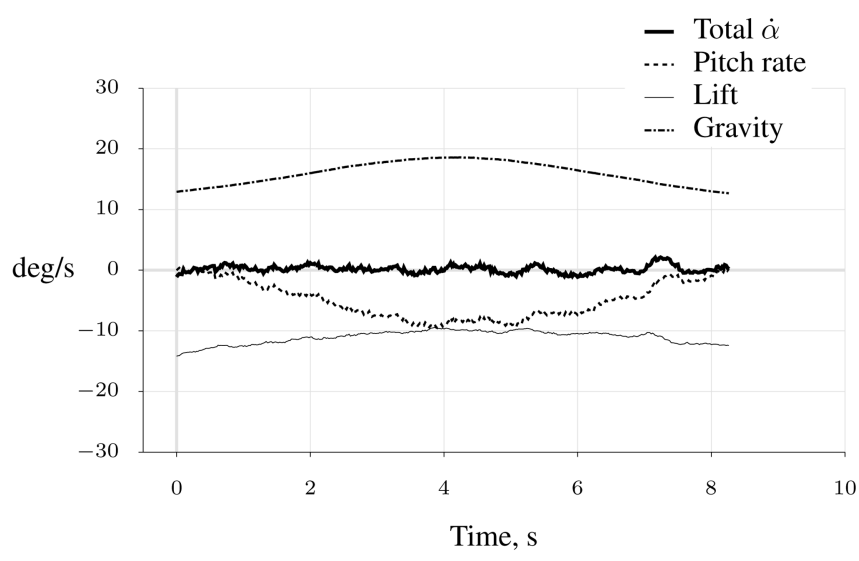

(b) Pushover maneuver

Figure 7.

Angle of attack rate decomposition, T-2 $q$-isolation maneuvers 
$\Delta V / V_{0}$

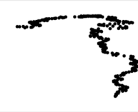

$-0.53$

$\Delta \alpha$

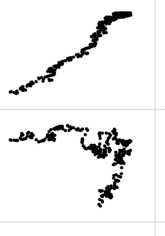

0.98

$-0.45$

$q \bar{c} / 2 V$

0.13

$-0.15$

0.15

$-0.76$

$-0.20$

$-0.69$

(a) Pullup maneuver

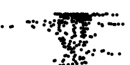

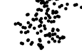

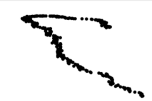

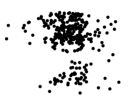
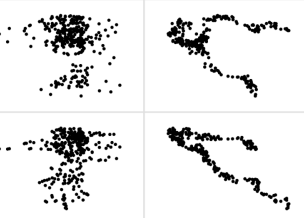

$\dot{\alpha} \bar{c} / 2 V$

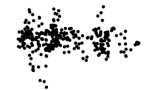

$\Delta \delta_{e}$

$-0.07$
$\Delta V / V_{0}$

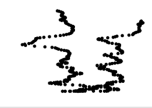

$-0.06$

$\Delta \alpha$

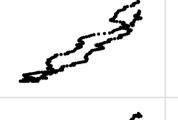
chere yor .. $. \cdot:$ :

$\therefore$ N. N

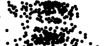

$\because \because$

0.95

0.14

$q \bar{c} / 2 V$

0.01

$-0.03$

$-0.85$

$-0.36$

(b) Pushover maneuver

Figure 8.

Regressor cross plots and correlation coefficients, T-2 $q$-isolation maneuvers 

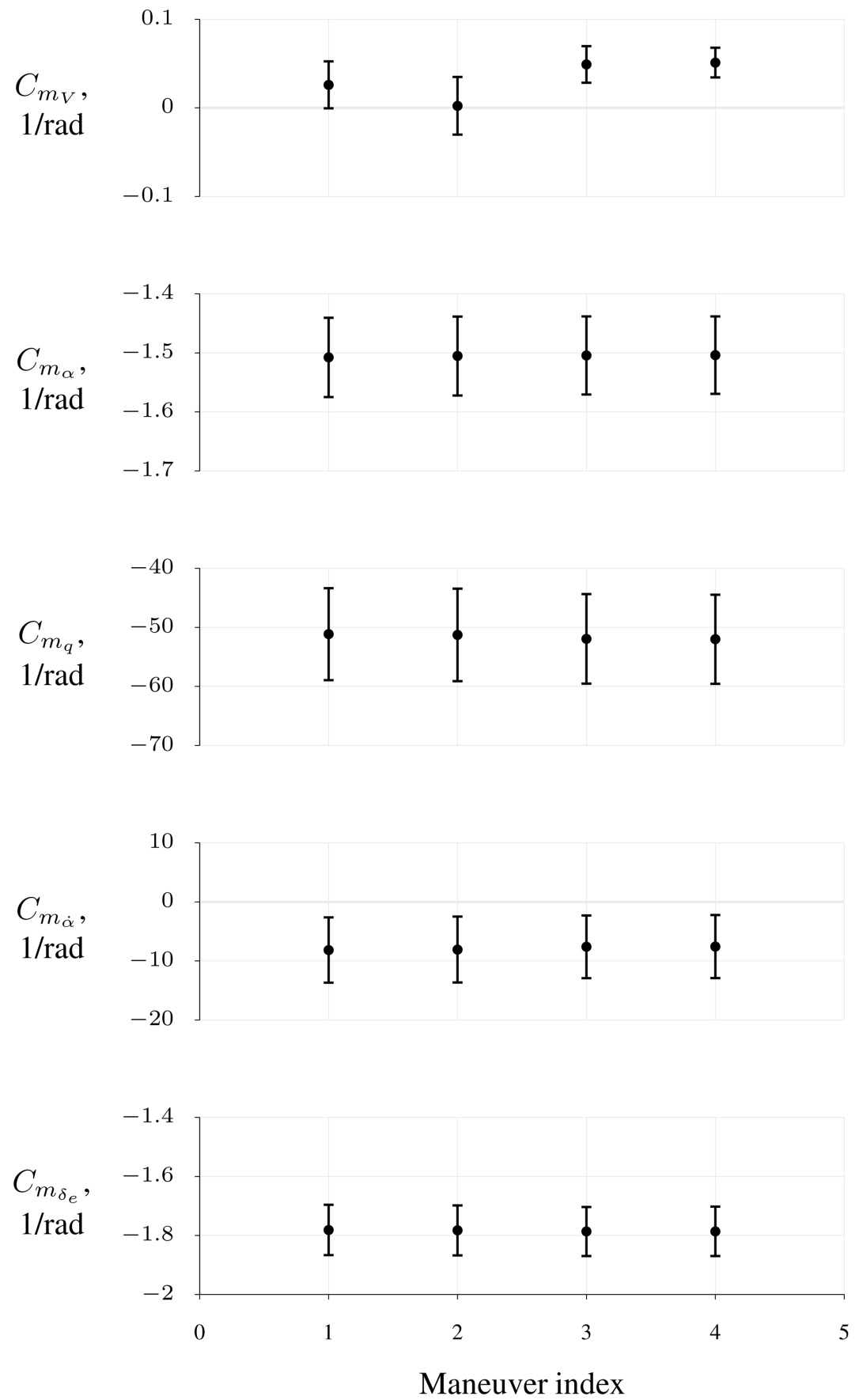

Figure 9.

Parameter estimates, T-2 straight and level combined with $q$-isolation maneuvers 


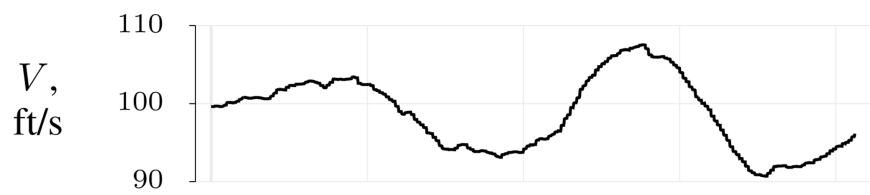

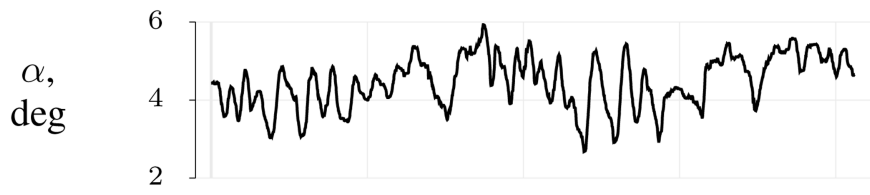
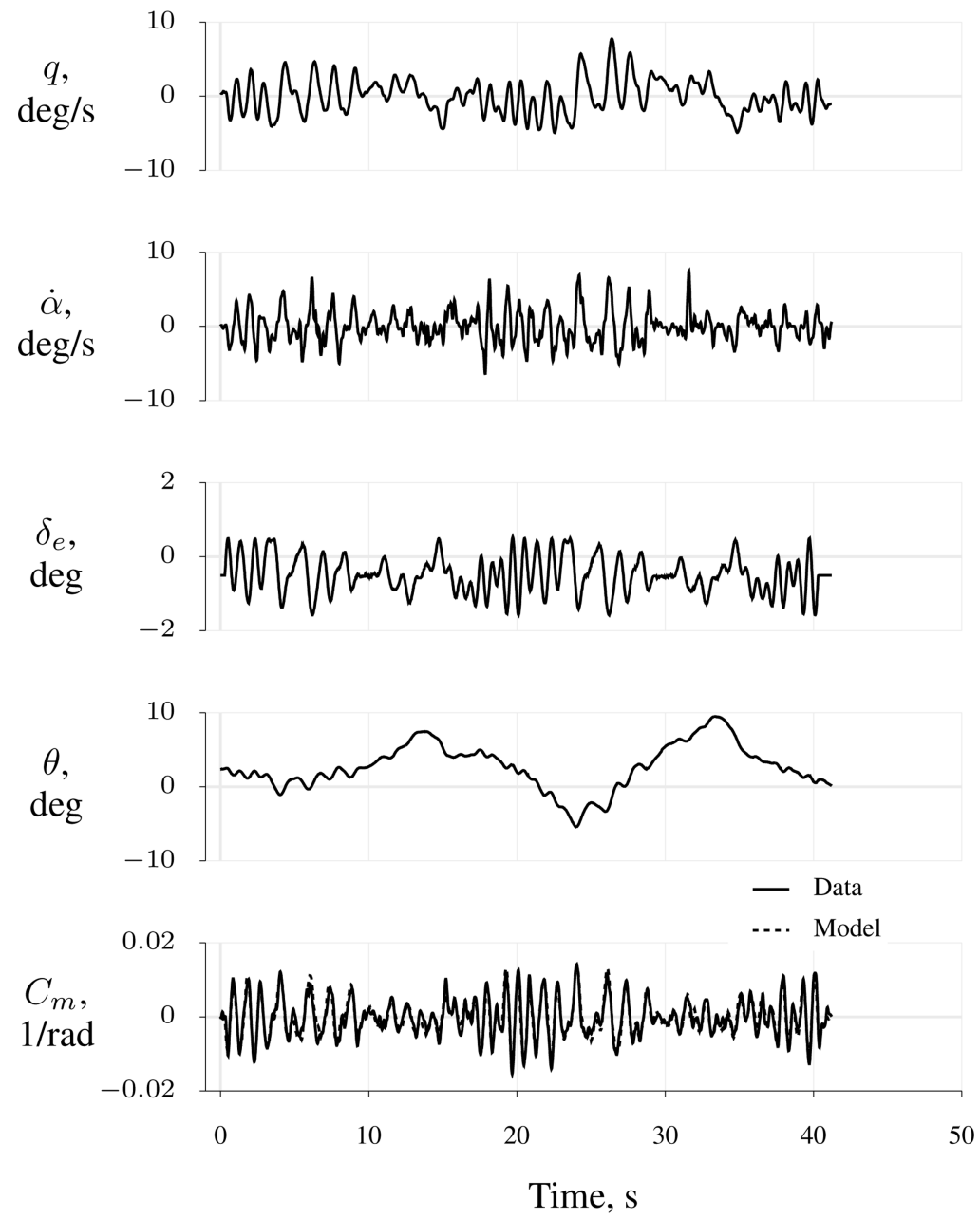

Figure 10.

Measurement time histories, Bat-4 multiple-mode maneuver 


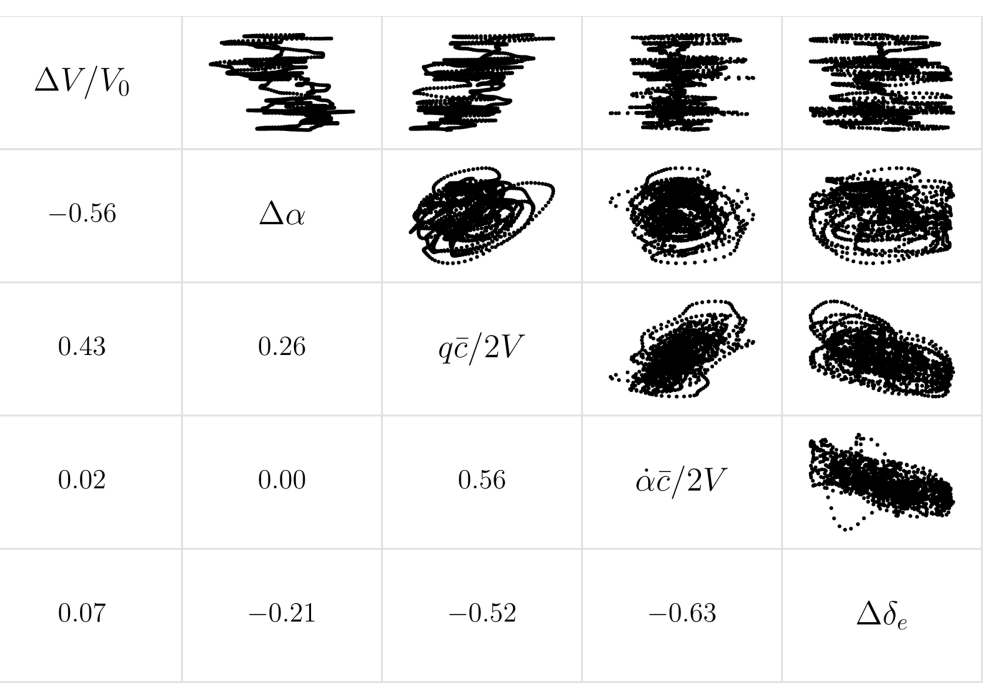

Figure 11.

Regressor cross plots and correlation coefficients, Bat-4 multiple-mode maneuver 


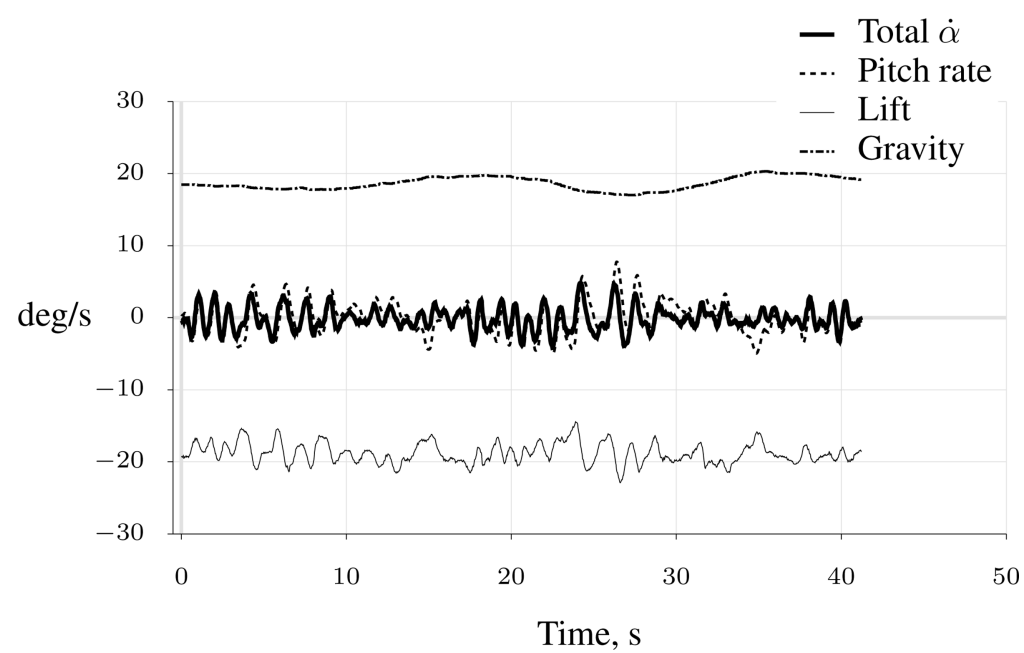

Figure 12.

Angle of attack rate decomposition, Bat-4 multiple-mode maneuver 

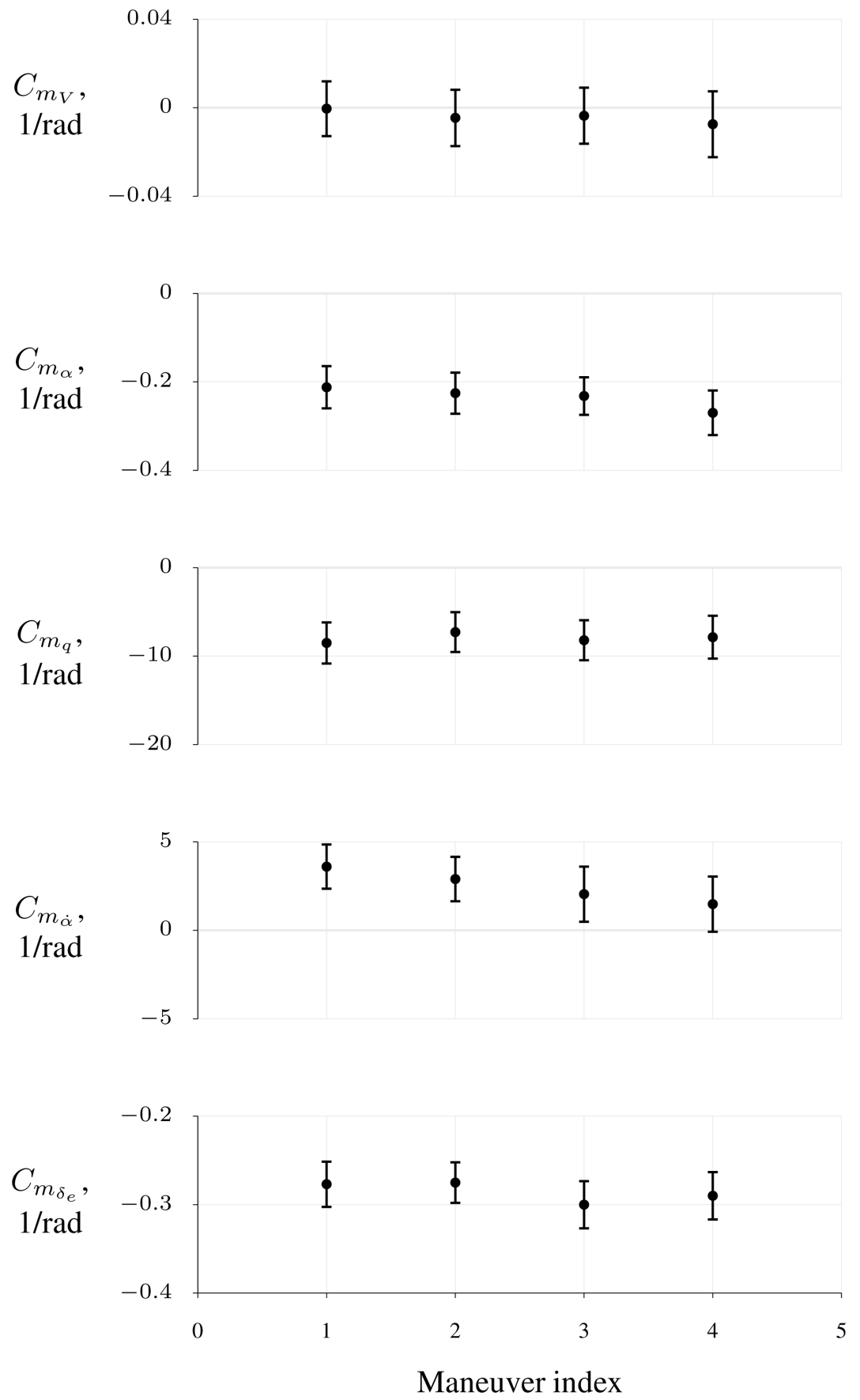

Figure 13.

Parameter estimates, Bat-4 multiple-mode maneuver 

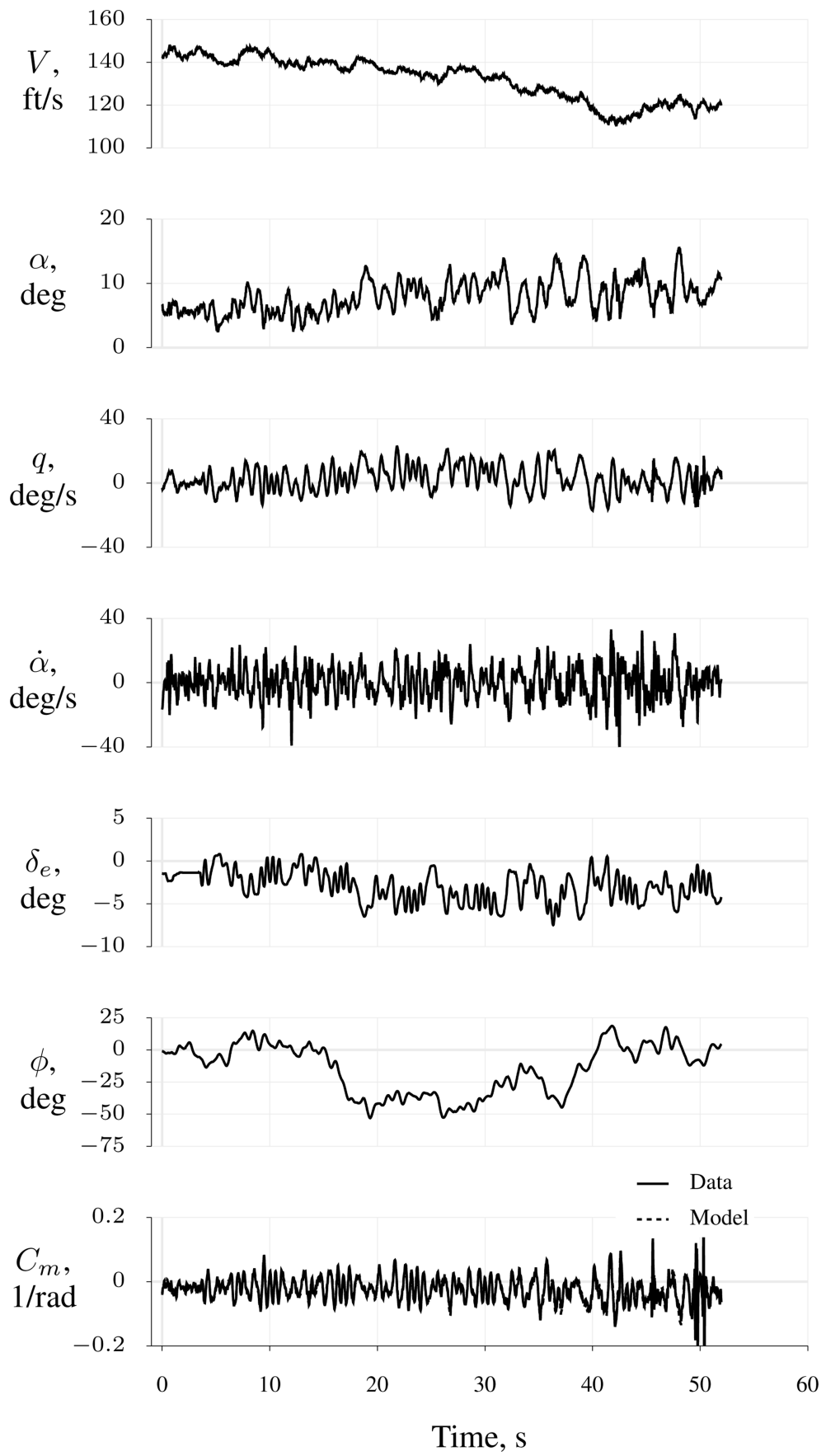

Figure 14.

Measurement time histories, T-2 turn excitation maneuver 
$\Delta V / V_{0}$
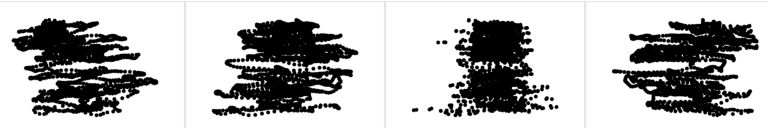

$-0.37$

$\Delta \alpha$
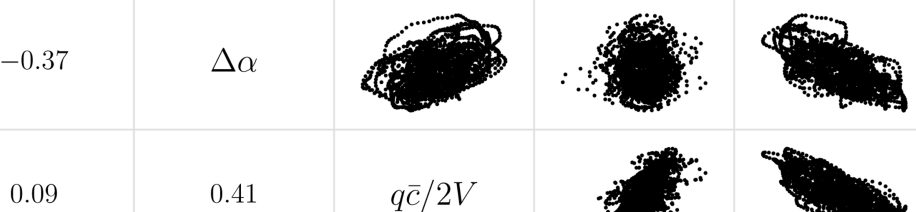

0.41

$q \bar{c} / 2 \mathrm{~V}$
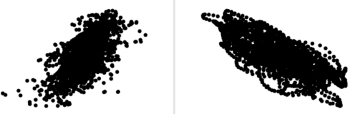

$-0.00$

0.00

0.64

$\dot{\alpha} \bar{c} / 2 V$

$-0.66$

$-0.76$

$-0.33$

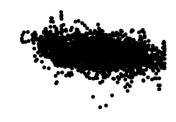

0.22

.

.7.

Figure 15.

Regressor cross plots and correlation coefficients, T-2 turn excitation maneuver 


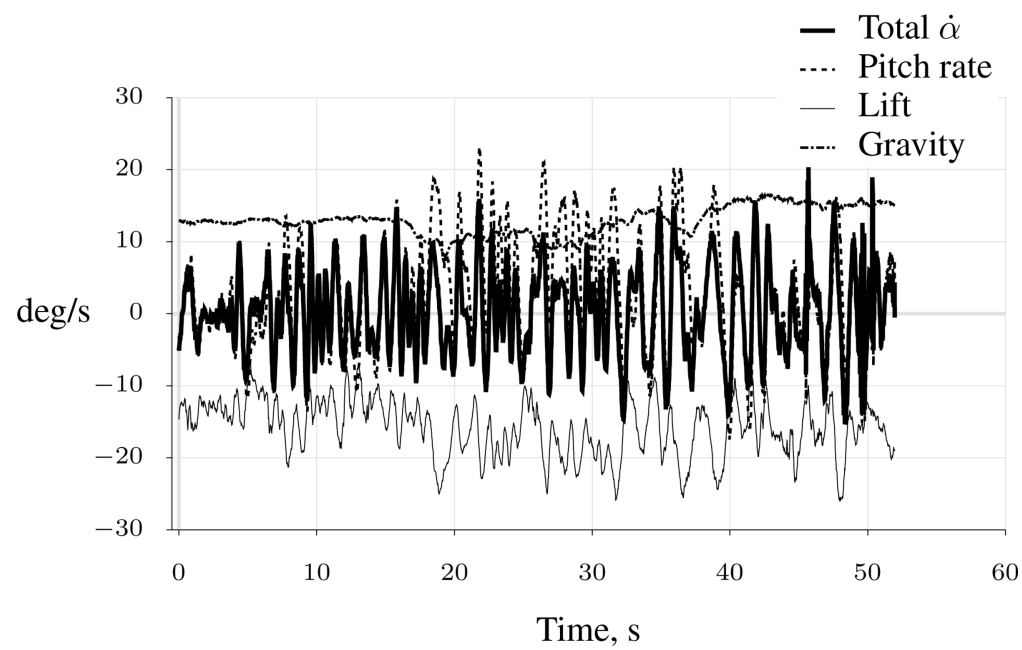

Figure 16.

Angle of attack rate decomposition, T-2 turn excitation maneuver

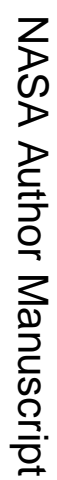



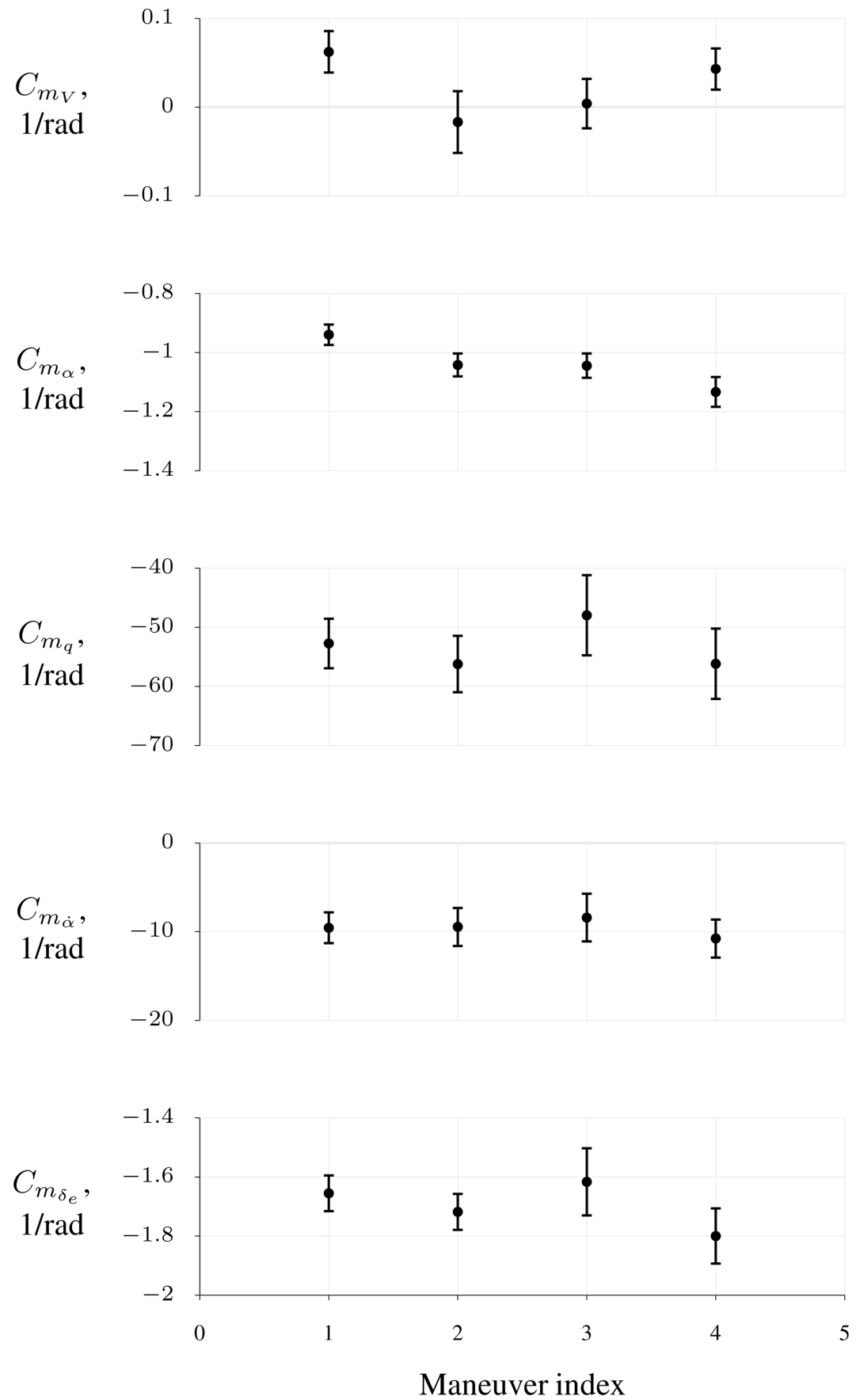

Figure 17.

Parameter estimates, T-2 turn excitation maneuver 
Table 1.

Test airplane geometry and nominal mass properties

\begin{tabular}{cccc}
\hline Parameter & Unit & T-2 & Bat-4 \\
\hline$b$ & $\mathrm{ft}$ & 6.849 & 12.68 \\
$\bar{c}$ & $\mathrm{ft}$ & 0.915 & 1.500 \\
$S$ & $\mathrm{ft}^{2}$ & 5.902 & 19.02 \\
$X_{c m}$ & in & 57.30 & 48.98 \\
$y_{c m}$ & in & 0.000 & 0.036 \\
$Z_{c m}$ & in & 11.43 & 24.01 \\
$m$ & slug & 1.585 & 3.593 \\
$I_{X X}$ & slug- $-\mathrm{ft}^{2}$ & 1.179 & 9.841 \\
$I_{y y}$ & slug- $-\mathrm{ft}^{2}$ & 4.520 & 8.861 \\
$I_{z z}$ & slug- $\mathrm{ft}^{2}$ & 5.527 & 16.17 \\
$I_{X z}$ & slug- $\mathrm{ft}^{2}$ & 0.211 & -1.015 \\
\hline
\end{tabular}

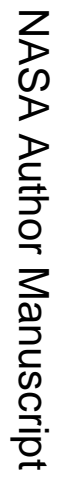

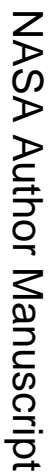


Table 2.

\begin{tabular}{|c|c|c|c|}
\hline & \multicolumn{3}{|c|}{ Parameter estimates and standard err } \\
\hline & Parameter & T-2 & Bat-4 \\
\hline & $C_{m_{V}}$ & $+0.05 \pm 0.01$ & $-0.01 \pm 0.00$ \\
\hline$\overline{\bar{\sigma}}$ & $C_{m_{\alpha}}$ & $-1.50 \pm 0.03$ & $-0.23 \pm 0.01$ \\
\hline$\sum$ & $C_{m_{q}}$ & $-52.6 \pm 3.24$ & $-7.96 \pm 0.58$ \\
\hline & $C_{m_{\dot{\alpha}}}$ & $-7.17 \pm 2.23$ & $+2.67 \pm 0.35$ \\
\hline$\overline{\widehat{N}}$ & $C_{m_{\delta_{e}}}$ & $-1.790 \pm 0.04$ & $-0.280 \pm 0.01$ \\
\hline
\end{tabular}

